\title{
Optimizing multiplex SNP-based data analysis for genotyping of Mycobacterium tuberculosis isolates
}

Sarah Sengstake ${ }^{1 *+}$, Nino Bablishvili ${ }^{2+}$, Anja Schuitema ${ }^{1}$, Nino Bzekalava ${ }^{2}$, Edgar Abadia ${ }^{3,4}$, Jessica de Beer ${ }^{5}$, Nona Tadumadze ${ }^{2}$, Maka Akhalaia ${ }^{6}$, Kiki Tuin $^{7}$, Nestani Tukvadze², Rusudan Aspindzelashvili², Elizabeta Bachiyska ${ }^{8}$, Stefan Panaiotov ${ }^{8}$, Christophe Sola ${ }^{4}$, Dick van Soolingen ${ }^{9,10}$, Paul Klatser ${ }^{1}$, Richard Anthony ${ }^{1}$ and Indra Bergval ${ }^{1}$

\begin{abstract}
Background: Multiplex ligation-dependent probe amplification (MLPA) is a powerful tool to identify genomic polymorphisms. We have previously developed a single nucleotide polymorphism (SNP) and large sequence polymorphisms (LSP)-based MLPA assay using a read out on a liquid bead array to screen for 47 genetic markers in the Mycobacterium tuberculosis genome. In our assay we obtain information regarding the Mycobacterium tuberculosis lineage and drug resistance simultaneously. Previously we called the presence or absence of a genotypic marker based on a threshold signal level. Here we present a more elaborate data analysis method to standardize and streamline the interpretation of data generated by MLPA. The new data analysis method also identifies intermediate signals in addition to classification of signals as positive and negative. Intermediate calls can be informative with respect to identifying the simultaneous presence of sensitive and resistant alleles or infection with multiple different Mycobacterium tuberculosis strains.
\end{abstract}

Results: To validate our analysis method 100 DNA isolates of Mycobacterium tuberculosis extracted from cultured patient material collected at the National TB Reference Laboratory of the National Center for Tuberculosis and Lung Diseases in Tbilisi, Republic of Georgia were tested by MLPA. The data generated were interpreted blindly and then compared to results obtained by reference methods. MLPA profiles containing intermediate calls are flagged for expert review whereas the majority of profiles, not containing intermediate calls, were called automatically. No intermediate signals were identified in 74/100 isolates and in the remaining 26 isolates at least one genetic marker produced an intermediate signal.

Conclusion: Based on excellent agreement with the reference methods we conclude that the new data analysis method performed well. The streamlined data processing and standardized data interpretation allows the comparison of the Mycobacterium tuberculosis MLPA results between different experiments. All together this will facilitate the implementation of the MLPA assay in different settings.

Keywords: Mycobacterium tuberculosis, MLPA, Data analysis, SNP typing, MAGPIX, Drug resistance, MTBC lineage, Republic of Georgia

\footnotetext{
*Correspondence: s.sengstake@kit.nl

${ }^{\dagger}$ Equal contributors

'KIT Biomedical Research, Royal Tropical Institute, Meibergdreef 39, 1105 AZ

Amsterdam, The Netherlands

Full list of author information is available at the end of the article
}

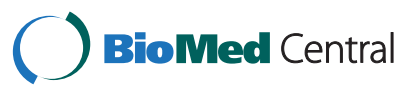

(c) 2014 Sengstake et al.; licensee BioMed Central Ltd. This is an Open Access article distributed under the terms of the Creative Commons Attribution License (http://creativecommons.org/licenses/by/4.0), which permits unrestricted use, distribution, and reproduction in any medium, provided the original work is properly credited. The Creative Commons Public Domain Dedication waiver (http://creativecommons.org/publicdomain/zero/1.0/) applies to the data made available in this article, unless otherwise stated. 


\section{Background}

Bacterial genotyping has a recognized potential to support tuberculosis (TB) control [1]. After initial diagnosis and before administered standardized empirical therapy timely detection of resistance mutations in the genome of Mycobacterium tuberculosis (MTB) can help clinical decision-making and support infection control efforts. Further, combined resistance and lineage identification is especially interesting in areas where there is a high prevalence of multidrug resistant TB (MDR-TB) and resistance is associated with specific lineages [2-4]. The association between lineage type, patient outcome, and drug resistance needs to be studied to improve future control measures. Unfortunately the benefits of strain identification are seldom optimally realized as mycobacterial genotyping, especially lineage identification, is almost always performed retrospectively on sampled isolates in high burden settings.

MTB evolves unidirectionally by the accumulation of mutations that are then fixed with no evidence of genetic exchange [5,6]. Drug resistance in MTB is almost exclusively conferred via SNPs [7]. Sets of SNPs and LSPs have also been identified as suitable markers for lineage identification within the MTB complex [8-10]. Consequently, SNP- and LSP-based assays are ideal for combined drug resistance testing and genotyping of MTB. We have previously developed and validated a bead-based multiplex ligation-dependent probe amplification (MLPA) assay and demonstrated its potential to simultaneously identify a range of drug resistance markers, discriminate within the genetic group MTB complex (MTBC), and detect and identify the clinically most relevant non-tuberculous mycobacterial species in cultured isolates [10-12].

MLPA assays or MLPA-like assays have been developed and reported by others for TB [20], other infectious agents [13-15], human DNA or RNA [16-18]. However, the beadbased tuberculosis-specific MLPA is unique in having a multiplexing capacity of up to 50 markers and combined detection of drug resistance and MTB lineage in a single assay. For high multiplexing assays a consistency check, i. e. the presence of sufficient markers and only markers exclusively from a single lineage, and streamlined data interpretation becomes increasingly useful and important as the numbers of markers screened increases. In previous reports we made use of a threshold value to classify fluorescent signals as positive or negative (see Figure four in, [10]). This threshold was based on preliminary testing of DNA from MTBC cultures and allowed accurate identification of the presence or absence of targeted markers in the majority of isolates tested [10]. However for signals that are close to the threshold automated interpretation of lineage types and drug resistance profiles can lead to a small proportion of unidentified false positives/false negatives which are not flagged as low confidence calls. As we perform a consistency check some of these calls will be identified, since many TB lineage markers are mutually exclusive, but for drug resistance markers this type of checking is not appropriate as mutations may occur in any lineage [19] and multiple mutations can be present in different combinations. To overcome this issue different approaches have been applied; for example adjusting the threshold value for all markers individually, making use of the signal-to-noise-ratio [15], reference genes [18], or calculating allelic ratios [20]. Here we have applied intrasample normalization and inter-sample correction to allow low confidence calls to be identified and flagged for scrutiny. Identifying intermediate signals allows only the high confidence calls to be classified as positive and negative. Automated interpretation for profiles with only high confidence calls can then be trusted without the need to be reviewed by an expert.

The Republic of Georgia is reported as one of the 18 high-priority countries in the WHO European Region with the highest TB burden [21]. Additionally in 2011, $15.8 \%$ of all diagnosed pulmonary TB patients were reported with at least MDR-TB of which $6.2 \%$ were infected with an extensively resistant TB strain. We collected samples from patients diagnosed with pulmonary $\mathrm{TB}$ at the National TB Reference Laboratory (NRL) of the National Center for Tuberculosis and Lung Diseases (NCTBLD) in Tbilisi, Georgia. In addition to smear microscopy and chest X-ray, phenotypic drug susceptibility testing (DST) and molecular drug resistance testing by GenoTypeMTBDRplus assay [22-24] is performed routinely in this setting leading to the continuous reporting of MTB surveillance data. In contrast there are presently no typing methods established in this setting and epidemiological data regarding TB lineage type are only assessed retrospectively outside of the country [25-27].

SNP typing can be complementary to whole genome sequencing (WGS) when applied to TB and other pathogens $[9,12,28-30]$. Whole genome high throughput sequencing is probably the ultimate technique for microbial genotyping but even sequencing a proportion of isolates evolving in a clonal population structure such as MTB and subsequent bioinformatic analysis [31] will reveal SNPs uniquely associated with specific genotypes and will contribute to the optimization and interpretation of SNP screening assays.

Here, we report a strategy that we have developed to analyze, visualize and interpret SNP-based data generated by MLPA while minimizing the required user input. We assess the degree to which the interpretation of the results such as resistance profile and MTB lineage can be confidently assigned.

\section{Results}

The principle of the new data analysis method and the calculation of the correction factors is described in detail 
in the Methods section and in Figure 1. The results obtained with the new data analysis method from DNA of cultured isolates from individual patient samples collected at the National TB Reference Laboratory in Tbilisi, Georgia is illustrated in Figure 2.

After MLPA data normalization, correction and automated calling, 4238/4300 (98.6\%) of the interrogated markers were classified as positive or negative (Figure 2). In total $62 / 4300(1.4 \%)$ markers were classified as intermediate. The 62 intermediate calls were not constraint to specific strains or specific targeted markers, which would indicate an underlying structural error in the analysis (see Additional file 1). In 11 of the 26 strains with intermediate calls these calls were confined to drug resistance associated markers. The remaining 15 isolates required expert interpretation for assignment to an MTB lineage. For 74 of the 100 analyzed isolates a full MLPA profile was obtained without review by an expert.

The results obtained from the MLPA and from the reference methods are summarized in Figure 3 and in Additional file 2. The MLPA produced interpretable profiles for 99 of the 100 isolates tested using the new data analysis method (Figure 3). The MLPA genotypes were called without user interpretation for 74 isolates, after expert review for a further 25 isolates and one isolate produced a profile that was uninterpretable.

To evaluate the accuracy of this calling strategy, all MLPA results were compared to results obtained from other methods. We used spoligotypes to validate the MLPA lineage types and the GenotypeMTBDRplus results as reference standard to validate the MLPA data obtained for the markers conferring resistance to first line drugs; DST was used as reference standard to validate the MLPA data obtained for the markers conferring resistance to second line drugs. In addition we have assessed the association between drug resistance and MLPA lineage (see Additional file 3) and MLVA clustering among all 100 isolates (see Additional file 4).

\section{Genotypic lineage identification}

The genotypes identified by MLPA and spoligotyping are compared in Table 1. The MTB4 lineage was the dominant genotype by MLPA (64/99) of which 16 MTB4 isolates were further classified as Latin American-Mediterranean (LAM) and eight as Haarlem. Beijing was the second most commonly identified lineage (35/99), within which the MLPA identified four sublineages (Table 1). These results were fully supported by spoligotyping for all but five isolates (Table 1).

MIRU-VNTR typing data was obtained for 87 samples of which two isolates (C39 and C67) showed multiple copy numbers in seven loci (see Additional file 2). Cluster analysis of the MLVA types revealed 33 isolates with non-

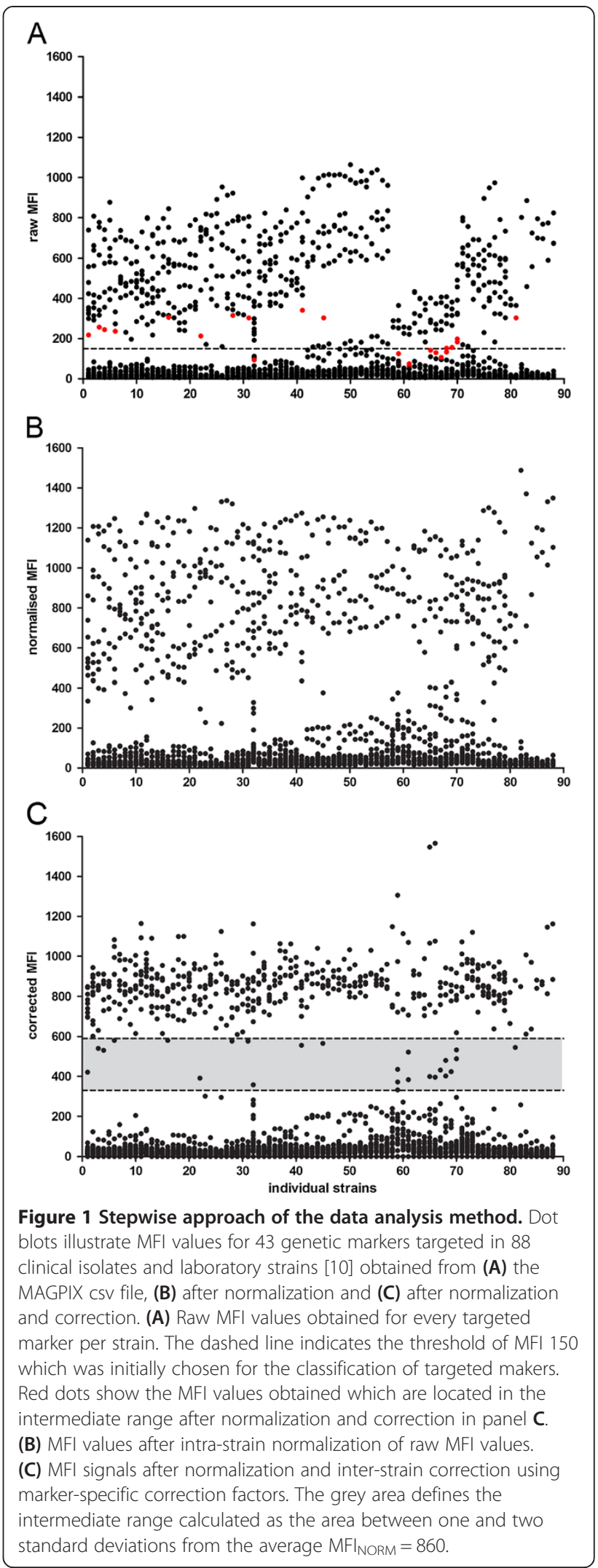




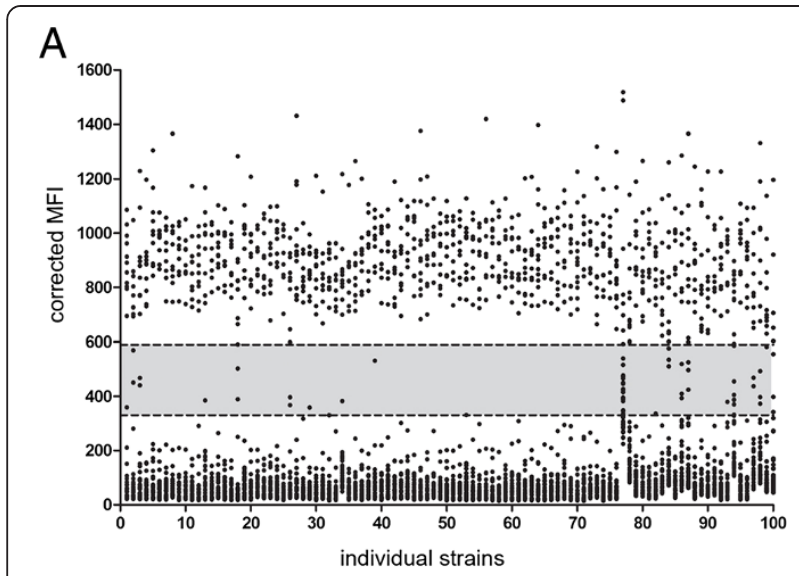

B

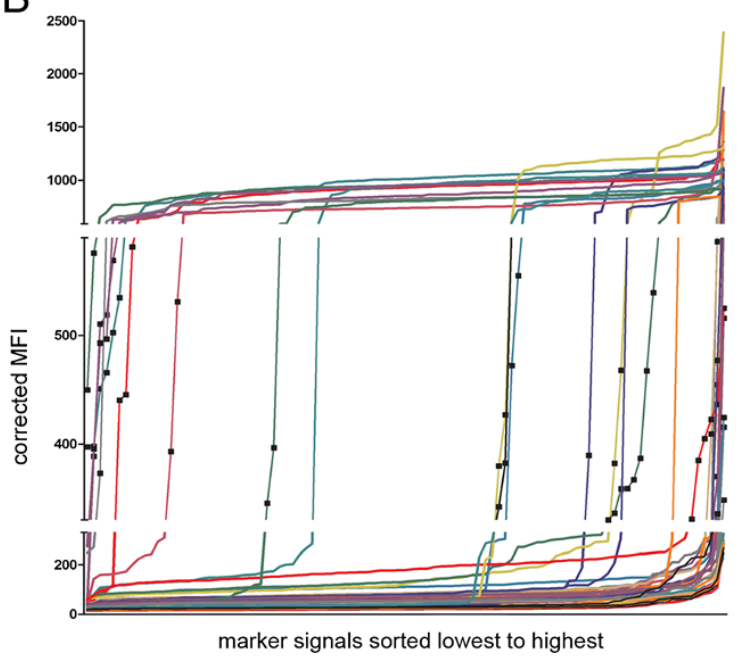

Figure 2 Visualization of data generated from 100 Georgian isolates after data normalization and data correction. (A) Dot plot showing normalized and corrected MFI values (black dots) per isolate for all 4300 markers targeted in the 100 Georgian isolates.

The grey area highlights $62(1.4 \%)$ unclassifiable markers of which 24 are drug resistance markers. Markers located above this area are classified as positive $(971,22.6 \%)$ and below as negative $(3267,76 \%)$. (B) Same data as shown in (A) but only the intermediate values are shown and visualized per marker. Each line shows the distribution of normalized and corrected MFI values, sorted from lowest o highest, (black squares), for one marker (individual colors).

unique MLVA types, forming 10 clusters (see Additional file 4).

\section{Drug resistance}

Results obtained from MLPA were compared to GenoTypeMTBDRplus (Table 2) and phenotypic DST results (see Additional file 5).

Of the 700 loci screened for rifampicin and isoniazid resistance ( $k$ at $\mathrm{G}$ codon 315 , inh A promoter region -15 , rpoB codons 176, 522, 526G and $526 \mathrm{~T}$ and 531) by MLPA in the 100 isolates, 500 could be compared to data obtained by the GenoTypeMTBDRplus assay (loci kat $\mathrm{G}$ codon 315, inh $\mathrm{A}$ promoter region -15 , rpoB codons 526G and $526 \mathrm{~T}$ and 531, the rpoB codons 176 and 522 could not be compared as they are only targeted by the MLPA assay. Results obtained by MLPA from 463 of the 500 (92.6\%) loci compared were directly supported by the GenoTypeMTBDRplus assay (Table 2). One isolate was identified as isoniazid resistant based on the loss of the katGWT1 probe by the GenoTypeMTBDRplus assay (Table 2) and no mutation was identified by MLPA suggesting the presence of a less common mutation in $k a t G$. For the remaining 37 loci no results were obtained by the GenoTypeMTBDRplus assay for three isolates (15 loci) and for 14 of the remaining 22 loci intermediate values were obtained by MLPA. For the remaining eight (1.6\%) loci there was a disagreement between the MLPA and the GenoTypeMTBDRplus assay.

The rpoB-S531L mutation was the only identified rifampicin resistance conferring mutation within the MDR-TB isolates (Table 2) both by MLPA and GenoTypeMTBDRplus. For three MDR isolates no mutation in $r p o B$ was identified by GenoTypeMTBDRplus or MLPA but the absence of rpoBWT probes shown by the GenoTypeMTBDRplus suggests the presence of a less common mutation in rpoB (Table 2).

Current probes included in the MLPA for second line drug testing, target mutations conferring resistance to the injectable drugs amikacin, kanamycin and capreomycin (rrs1401), capreomycin (rrs1402) or fluoroquinolones (gyrA90 and gyrA94). MLPA identified resistance to amikacin, kanamycin and capreomycin in two of six MDR-TB isolates resistant to kanamycin and capreomycin by DST (see Additional file 5). Two isolates showed resistance to ofloxacin by DST, but no fluoroquinolone resistance was detected by MLPA in any of the 100 isolates.

DST identified 16 isolates with ethambutol resistance. A mutation in the 306 codon of the $e m b B$ gene conferring resistance to ethambutol was confidently detected by MLPA in only four isolates all of which were also phenotypically resistant (see Additional file 5). Additionally MLPA detected an intermediate value for ethambutol resistance in two isolates one of which was determined ethambutol resistant by DST (see Additional file 5). MLPA identified a mutation in the codon 43 of the rpsL gene conferring resistance to streptomycin in 11 of 52 phenotypically streptomycin resistant isolates $(25 \%)$ and in one the $44(2.2 \%)$ phenotypically sensitive isolates. In one of the 52 isolates that were phenotypically streptomycin resistant an intermediate signal for the rpsl-43 marker was obtained.

\section{Discussion}

We believe that an easily interpretable, robust, highthroughput multiplex genotyping method such as MLPA can support infectious disease monitoring and control. 


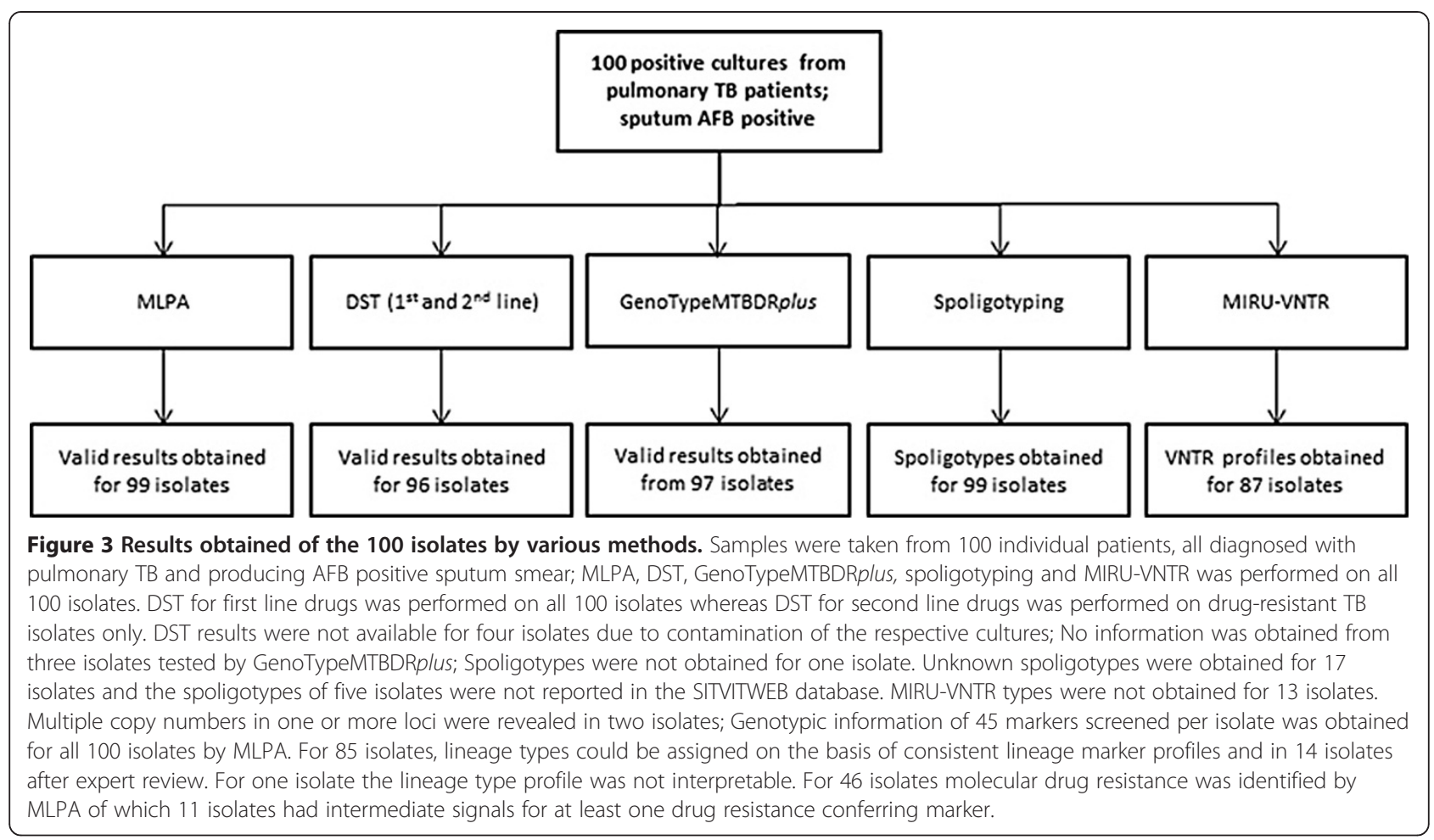

Critically unlike many typing methods SNP-based genotyping is directly compatible with data generated by WGS/next generation sequencing (NGS). Synergy between SNP-based surveillance and SNP discovery could be facilitated by cost-effective high throughput assays. Moreover, routine screening of clinical isolates with WGS is currently not feasible in most high prevalence MDR-TB settings, while a robust and dedicated method like MLPA is more practical to implement. Also, the read out platform used here, the Luminex technology is relatively robust and suitable for other assays notably the newly developed TB-SPRINT method [32].

As a result of the clonal population structure of the MTB complex, genotypic information such as SNPs and large sequence polymorphisms that are not under strong selective pressure can be used to identify specific genotypic lineages and are unique and mutually exclusive. Indeed, many possible MLPA profiles are invalid, as MTB is clonal, a pure MTB strain will contain all and only genetic markers from a single lineage $[8,9]$. This allows an internal quality check, unlike classical typing methods for example IS6110 RFLP typing, or VNTR typing and to some extent spoligotyping [33-35] where virtually all profiles are theoretically possible.

Here we have analysed a panel of 100 isolates from patients diagnosed with pulmonary $\mathrm{TB}$ and with positive sputum smear microscopy collected in 2011 at the NCTBLD in Tbilisi, Georgia and used the data generated to evaluate a novel data analysis strategy. From the 100 isolates analyzed, 74 were confidently assigned to an MTBC lineage by our algorithm without expert interpretation. After expert review of the remaining 26 MLPA profiles only a single profile remained uninterpretable. The results of these 99 profiles were compared to reference methods.

Previously we called positives on the basis of a threshold MFI (equal or higher than 150) with no intermediate signals [10]. Analyzing the data presented here using the threshold method would have resulted in 951 of the 4300 characteristics being called positive and 3349 negative. Using our new data analysis method 971 characteristics were called positive and 3267 called negative, 62 MFI signals were classified as intermediate. The 62 intermediate calls were distributed amongst 26 isolates, and 23 of the $62(37 \%)$ were markers related to drug resistance distributed amongst 11 isolates. Of these 62 intermediate calls 13 would have been called positive by the original analysis method (four correctly, seven incorrectly and two unknown due to the lack of a reference standard) and 49 negative (21 correctly, 10 incorrectly, 18 unknown). With respect to lineage identification the threshold method would have resulted in 22 inconsistent profiles in contrast to 14 profiles flagged for expert review (of which 13 could be resolved) with the new data analysis method.

Importantly, as we normalize with pre-established marker correction factors a single profile can be interpreted and no minimum sample size is required to validate the calls in contrast to other approaches [36]. Those 
Lineage type by spoligotyping

Lineage type by MLPA Beijing H1 H3 LAM1 LAM5 LAM9 T5-RUS1 T5-RUS1 MANU2 T1 T1-RUS2 T2 T3 T4 T5 Ural-1 ${ }^{\mathrm{b}}$ Unknown Unknown Not reported Not-

interpretable

\begin{tabular}{|c|c|c|c|c|c|c|c|c|c|c|c|c|c|c|c|c|c|c|c|c|}
\hline & & & & & & & & & & & & & & & & & & & & \\
\hline Beijing K1 ( $n=28)$ & 28 & & & & & & & & & & & & & & & & & & & \\
\hline Beijing $V+/ C H I N+(n=5)$ & 5 & & & & & & & & & & & & & & & & & & & \\
\hline Beijing SA-/CHIN- $(n=1)$ & 1 & & & & & & & & & & & & & & & & & & & \\
\hline Beijing $\vee-(n=1)$ & & & & & & & & & & & & & & & & & 1 & & & \\
\hline $\begin{array}{l}\text { MTB4 other than LAM or } \\
\text { Haarlem }(n=40)\end{array}$ & & & 1 & & & & & & 1 & 8 & 1 & & 1 & 1 & 1 & 9 & 13 & & 4 & \\
\hline $\operatorname{LAM}(n=16)$ & & & & 3 & 1 & 1 & 2 & 3 & & & & 1 & & & & & 3 & 1 & 1 & \\
\hline Haarlem $(n=8)$ & & 2 & 6 & & & & & & & & & & & & & & & & & \\
\hline Non-interpretable $(n=1)$ & & & & & & & & & & & & & & & & & & & & 1 \\
\hline Total (100 isolates) & 34 & 2 & 7 & 3 & 1 & 1 & 2 & 3 & 1 & 8 & 1 & 1 & 1 & 1 & 1 & 9 & 17 & 1 & 5 & 1 \\
\hline
\end{tabular}

Lineage types were obtained from spoligotyping using the SITVITWEB database. a, isolates were identified as T5-RUS1 or Unknown according to SITVITWEB but LAM according to their spoligotype identification type [41]; b, isolates were identified as Ural-1 on the basis of their SIT according to [42]; Numbers in bold indicate disagreement between MLPA and spoligotyping. 
Table 2 Correlation between drug resistance identified by MLPA and GenoTypeMTBDRplus

\begin{tabular}{|c|c|c|c|c|c|c|c|c|c|c|}
\hline \multirow[b]{2}{*}{$\begin{array}{l}\text { Drug resistance by } \\
\text { MLPA }\end{array}$} & \multicolumn{10}{|c|}{ Drug resistance by GenoTypeMTBDRplus } \\
\hline & rpoBWT & rpoBMUT3 & $\begin{array}{c}\text { rpoBMUT } \\
\text { other }^{\mathrm{a}}\end{array}$ & katGWT & katGMUT1 & $\begin{array}{c}\text { katGMUT } \\
\text { other }^{\mathbf{b}}\end{array}$ & inhAWT & inhAMUT1 & $\begin{array}{c}\text { inhAMUT1 and } \\
\text { katGMUT1 }\end{array}$ & No result \\
\hline no rpoB mutation & 76 & 0 & 3 & & & & & & & 2 \\
\hline intermediate rpoB & 6 & 1 & 0 & & & & & & & 1 \\
\hline rpoB-S531L (rpoBMUT3) & 0 & 11 & 0 & & & & & & & 0 \\
\hline rpoB-S522L & 1 & 0 & 0 & & & & & & & 0 \\
\hline no katG mutation & & & & 74 & 0 & 0 & & & & 1 \\
\hline katG-S315T (katGMUT1) & & & & 0 & 14 & 1 & & & & 1 \\
\hline intermediate katG & & & & 1 & 0 & 0 & & & & 0 \\
\hline no inhA mutation & & & & & & & 64 & 0 & 0 & 2 \\
\hline inhA-15 C/T (inhAMUT1) & & & & & & & 6 & 2 & 0 & 0 \\
\hline inhAMUT1 and katGMUT1 & & & & & & & 0 & 0 & 5 & 1 \\
\hline intermediate inhA & & & & & & & 5 & 0 & 0 & 0 \\
\hline $\begin{array}{l}\text { Total isolates } \\
\text { (GenoTypeMTBDRplus) }\end{array}$ & 82 & 12 & 3 & 75 & 14 & 1 & 75 & 2 & 5 & 3 \\
\hline
\end{tabular}

Mutations obtained by MLPA are indicated for the respective genetic marker investigated with the GenoTypeMTBDRplus assay; isolates for which no results were obtained by GenoTypeMTBDRplus MLPA results for all genetic markers are indicated; an intermediate result indicates the presence of an intermediate signal and thus no presence or absence for the respective genetic marker; $a=$ two isolates did not show a positive band with the rpoBWT8 probe but no mutation probe was positive; one isolate did not show a positive band for the rpoBWT3 and rpoBWT4. $b=$ one isolate did not show a positive band with the katGWT1 probe but no mutation probe was identified.

profiles containing of intermediate signals or those with a profile inconsistent with established lineage identification are flagged and need to be inspected by an expert. This approach means that samples with poor DNA quality, experimental error or experimental variability are identified and not spuriously classified automatically. Figure $2 \mathrm{~B}$ shows that for each marker, when the MFI values are ordered from lowest to highest, for the 100 isolates analyzed each marker ideally follows a sigmoid curve with only very few values in the intermediate range - these are the intermediate calls.

In the Georgian region a diverse phylogeny of MTB has been previously observed [25,26,37]. Genotyping by MIRU-VNTR, spoligotyping and MLPA of 100 randomly sampled isolates from this region here supports this diversity as well as allowing us to validate our assay by comparing the genotypes obtained by each method. Spoligotypes were obtained for 99 of the 100 isolates of which 17 were unknown (ambiguous) in the SITVITWEB database and five were not previously reported (Table 1). For the 77 isolates for which an MLPA profile and known spoligotype was available $72(93.5 \%)$ matched. The one isolate with no MLPA profile available was "direct repeats" negative by spoligotyping. The 22 isolates with unknown or not previously reported spoligotypes were all classified to a specific lineage type by MLPA (Table 1) these classifications were supported by MIRU-VNTR (see Additional file 2).

Genetic markers under strong selective pressure, most notably antimicrobial resistance associated mutations, may occur in multiple lineages [19]. Some lineages have been suggested to be more associated with (multi-) drug resistance and therefore identification of some lineages may be particularly important for public health (reviewed in [1]). Data collected between 2003 and 2005 in the WHO European Region and elsewhere shows that majority of MDR-TB isolates belonged to the Beijing lineage [2]. An association of the Beijing lineage with multi-drug resistance has previously been also observed in Georgia [25-27] and in the study presented here a member of the Beijing lineage was $7.4 \times(37 \%$ versus $5 \%)$ more likely to also have an MDR profile than members from other lineages. In order to confirm these findings testing larger numbers of epidemiologically more representative sample sets needs to be undertaken. Methods such as MLPA are suitable to undertake these studies; for example even in this limited initial study the Beijing clone K1, MLVA type 94-937 was for the first time identified in Georgia for four isolates two of which were MDR (see Additional file 4).

Intermediate calls might indicate the presence of sensitive and resistant alleles. One isolate that was MDR-TB by GenotypeMTBDRplus had an intermediate signal for rpoB-531 and embB306 by MLPA after data analysis, possibly indicating heteroresistance or mixed infection. Further evaluation of the data analysis method is required to prove the ability of the current MLPA to detect heteroresistance. The acquisition of resistance during MDR-TB therapy has also been documented, as was detected by MLPA, in South Africa and shown to affect the performance of molecular assays that detect resistance to second line drugs [38]. Two studies report the occurrence of drug 
resistance amplification and patients infected with more than one genotype in Georgia [27,39]. Resistance amplification and re-infection with MDR-TB isolates could be partially accountable for the high failure rates in especially new TB patients in Georgia [21].

At present a limitation of the MLPA assay is that it is only suitable for use on cultured isolates but developments in real time assays appear to provide the possibility to perform an MLPA assay in a closed tube format [40] which could dramatically simplify implementation.

\section{Conclusions}

At the NCTBLD in Tbilisi, Georgia, no method for extensive genotyping or strain identification was implemented prior to this work. Therefore, lineage identification and phylogenetic analysis need to be performed outside of the NCTBLD. As a result of this study, the development of a transparent analysis algorithm, and associated staff training the MLPA method is now able to be performed on site and a report of the first year's data is in preparation. Even when performed locally on cultures, rather than directly on sputum, combined lineage identification and screening for drug resistance can provide much needed insight into the genotypic background of circulating drug resistant strains in a more timely manner.

\section{Methods}

\section{Bacterial/DNA samples}

Positive cultures from 100 individual patients referred to the NRL, Tbilisi, Georgia were collected. Isolates were randomly selected from patient samples between January and April 2011 with a positive acid-fast bacilli (AFB) microscopy result and diagnosed with pulmonary TB; 28 were from patients previously treated for TB. Ethical approval was not required for this study as no patient information was used and the results of the analysis of the bacterial cultures could not be linked back to individual patients.

Within the routine workflow for TB patient sample testing at the NRL the following data was collected: Sputum samples were directly inoculated onto LJ -based solid medium and BACTEC MGIT 960 system (BD, Sparks, MD, USA) drug susceptibility testing (DST) against first-line drugs for isoniazid and rifampicin was performed using the absolute proportion method on Löwenstein-Jensen (LJ) medium or the BACTEC MGIT 960 system [23] and molecular drug resistance testing for isoniazid and rifampicin using the Genotype MTBDRplus assay (Hain Lifescience, Nehren, Germany) [22]. Routine Genotype MTBDRplus assay results were obtained directly from DNA extracted from sputum only if the sputum smear was positive and the sample was no older than four days, otherwise MTBDRplus was performed on subsequent positive cultures. All isolates identified as drug-resistant-TB were additionally subjected to second line DST (proportion method on LJ medium) [23]. DST against the second-line drugs ethionamide (Eto), ofloxacin (Ofx), para-amino-salicylic acid (PAS), capreomycin $(\mathrm{Cm})$ and kanamycin $(\mathrm{Km})$ was performed using the proportion method on LJ medium as previously described [23].

DNA extracted for routine GenoTypeMTBDRplus testing was also used for MLPA analysis. In brief, $1 \mathrm{ml}$ bacterial culture was taken from a respective BACTEC MGIT culture. Bacterial cells were pelleted and resuspended in $100 \mu \mathrm{l}$ molecular grade water. DNA was released by thermolysis at $95^{\circ}$ Celsius for $30 \mathrm{~min}$ and sonication for $15 \mathrm{~min}$. Cell debris was pelleted and the supernatant containing the DNA was stored at $-20^{\circ}$ Celsius. MLPA analysis was performed, as explained below, without any knowledge of the routine test results at KIT Biomedical Research (Royal Tropical Institute), Amsterdam.

\section{MLPA}

The bead-based MLPA was performed as previously described with an identical 43-plex probe mix [10] on a MAGPIX ${ }^{\circledast}$ device (Luminex Corp., Austin, Texas, USA). In brief, 13 drug resistance markers and 30 phylogenetic informative markers were targeted in the mycobacterial genome and two controls LumH and LumD were run with every sample. For quality control of the bead-based MLPA four control samples are run with every MLPA experiment [10]: assay control, no template control, contamination control and MAGPIX control. Molecular markers associated with resistance to streptomycin (S), isoniazid (I), rifampicin (R), and ethambutol (E) are included targeting respectively the wildtype allele in the rpsl-43 locus, katG (AGC $315 \mathrm{ACC})$ and inhA (-15C/T), rpoB (GTC 176 TTC), rpoB (TCG 522 TTG), rpoB (CAC $526 \mathrm{GAC}$ ), rроB (CAC $526 \mathrm{TAC}$ ), rроB (TCG 531 TTG) and the wildtype allele in the $e m b B 306$ locus. Mutations associated with molecular resistance to the injectable drugs amikacin (Am), kanamycin $(\mathrm{Km})$ and capreomycin $(\mathrm{Cm})$ are also targeted by inclusion of probes for the wildtype allele in rrs (1401) (Am, Km, Cm) and rrs $(1402 \mathrm{C} / \mathrm{T})(\mathrm{Cm})$. The fluoroquinolone (FLQ) resistance associated mutations gyrA (GCG 90 GTG) and gyrA (GAC 94 GGC) were also targeted.

The assignment to lineages within the MTB complex was based on the previously published algorithm [10]) and comprehensive MTB complex phylogeny [8-10]. A slightly modified version of the algorithm proposed in [10] was used for the identification of MTB lineages and sublineages (see Additional file 6). The markers RD-seal and RD1-mic were added for the identification of Mycobacterium microti or Mycobacterium pinnepedii, respectively and the algorithm for identification of non-tuberculous 
mycobacteria was removed. Raw MFI signals obtained for all samples from the MAGPIX csv-file are compiled in Additional file 7.

\section{MLPA data analysis}

The csv-file produced after every experiment by the Luminex xPonent 4.2 software was imported into an Excel worksheet (Microsoft, Redmond, USA). First an intra-sample normalization (1. below) on the raw Median Fluorescence Intensity (MFI) signals was performed to allow inter-sample comparisons. This was followed by the application of marker-specific correction factors (2. below), which simplifies interpretation and visualization of the results. The MFI signals from the controls LumD and LumH were excluded from the data normalization process.

\section{Calculation of $\mathrm{MFI}_{\mathrm{NORM}}$.}

On the basis of the currently used MLPA probes and an optimally performing MLPA assay, at least five markers will give a true positive signal in every MTB isolate with a good quality DNA independent of the drug resistance profile or lineage type. The normalized MFI value $\left(\mathrm{MFI}_{\mathrm{NORM}}\right)$ represents the raw MFI signal (Figure 1A) of each probe per sample of a run multiplied by an arbitrary factor (5000) and divided by the sum of the first five highest MFI signals per isolate (Figure 1B). The value of 5000 as numerator was chosen to facilitate visualization of the data only. It does not qualitatively influence the data results.

$$
M F I_{\text {Norm }}=\frac{M F I_{\text {RAW }} \times 5000}{\text { sum first five highest MFI signals per isolate }}
$$

\section{Calculation of $\mathrm{MFI}_{\mathrm{CORR}}$}

To ease interpretation and allow a single cut-off to be applied for each marker all normalized MFI values were multiplied with previously calculated marker-specific correction factors.

\section{Calculation of marker-specific correction factors}

For the calculation of marker-specific correction factors previously published data from 88 well characterized laboratory strains and clinical isolates [10] were normalized as in (i) above. Then the average $\mathrm{MFI}_{\mathrm{NORM}}$ was calculated for each marker (e.g. inhA-15) using only the true positive signals of the marker. The overall average $\mathrm{MFI}_{\mathrm{NORM}}$ was calculated subsequently by adding all marker-specific $\mathrm{MFI}_{\mathrm{NORM}}$ averages and dividing by the number of all markers. The average $\mathrm{MFI}_{\text {NORM }}$ was 850 .
We also calculated +1 and -1 standard deviation (SD) of the average $\mathrm{MFI}_{\mathrm{NORM}}$ (average $\mathrm{MFI}_{\mathrm{NORM}}=850,1^{\text {st }} \mathrm{SD}+/$ 260). The area of low mathematical confidence calls was determined to be between -1 and -2 SD from the average of all positives (MFI $\mathrm{CORR} 590$ - 330) (Figure 1C, grey area). Marker-specific correction factors were calculated by dividing the overall average $\mathrm{MFI}_{\mathrm{NORM}}$ (value of 850 ) with the marker-specific average $\mathrm{MFI}_{\mathrm{NORM}}$.

All MFI $_{\text {NORM }}$ values of a new dataset, here the 100 isolates, were multiplied with the calculated standard marker-specific correction factors calculated above resulting in the corrected MFI ( $\left.\mathrm{MFI}_{\mathrm{CORR}}\right)$ (Figure 2A-B). Visualization of the raw data with respect to isolate (Figure 2A) and marker (Figure 2B) is shown. Calling of the results: For each isolate analyzed the genotype was called if no intermediate genotypic markers were present or flagged for final interpretation by an expert if the MFICORR of one or more genotypic markers was in the grey area (intermediate). Thus genotype profiles were called without user interpretation, resolved by an expert or identified as un-interpretable by an expert.

\section{Spoligotyping}

Spoligotyping was performed at the University Paris-Sud on a Luminex $200^{\circ}$ and/or on the MAGPIX ${ }^{\circ}$ platform on all 100 samples using 43 spacer-spoligotyping [34] on an aliquot of the same DNA sample used for the MLPA analysis. Spoligo International Type (SIT) numbers and lineages were assigned according to the SITVITWEB database [33] and adapted according to [41,42]. Octal and binary codes reported in the SITVITWEB database that do not provide lineage information are referred to as 'unknown'. Octal and binary codes that are not identified in the SITVITWEB database are referred to as 'not reported before'.

\section{MIRU-VNTR typing}

Standard VNTR typing using 24 loci was performed as previously described by Supply et al. [43] and optimized by the RIVM [44] at the RIVM in Bilthoven. Identification of MLVA 15-9 codes was carried out by using the MIRU-VNTRplus database [45]. A cluster was defined as a minimum of two isolates with identical MIRU-VNTR patterns.

\section{Statistics}

A One-tailed Fisher's exact test was used to statistically assess if the MDR phenotype was equally distributed amongst the genotypes Beijing and non-Beijing. The P-value was calculated using a two-by-two table with Beijing and MDR (13 isolates), Beijing and non-MDR (21 isolates), non-Beijing and MDR (3 isolates), nonBeijing and non-MDR (58 isolates). 


\section{Additional files}

\author{
Additional file 1: Table S1. Intermediate calls stratified per individual
} marker.

Additional file 2: Table S2. Summary of data obtained for 100 isolates. Additional file 3: Table S3. DST results for 100 Georgian isolates stratified according to the MLPA lineage type.

Additional file 4: Table S4. MLVA clusters identified in 100 Georgian isolates.

Additional file 5: Table S5. Correlation between drug resistance identified by MLPA and DST.

Additional file 6: Algorithm applied to all isolates analyzed for lineage identification of Mycobacterium tuberculosis complex. MLPA markers are framed and final MTB complex lineages or sublineages are shown in bold. The species identification of a sample starts with the MTB complex 16SrRNA marker. As an example the call for the Beijing lineage $\mathrm{K} 1$ is highlighted with bold arrows. The following markers are present or absent in an isolate belonging to the Beijing K1 lineage: MTBC 165 rRNA (present), TbD1 (present), RD750 (RD sequence present), pks15/1-7 (absent), RD105 (RD sequence deleted), fbpB-238 (present), mutT2-58 (present), acs-1551 (absent), RD131 (RD sequence deleted). MTBC, MTB complex. EAl, East African Indian; CAS, Central Asian; LAM, Latin American Mediterranean; Updated version of [10]

Additional file 7: Table S7. Raw MFI data of the MLPA assay.

\section{Abbreviations}

TB: Tuberculosis; MTBC: Mycobacterium tuberculosis complex; SNP: single nucleotide polymorphism; MDR: Multidrug resistance; DNA: Deoxyribonucleic acid; MLPA: Multiplex ligation-dependent probe amplification; DST: Drug susceptibility testing; MIRU-VNTR: Multiple interspersed receptive units - variable number of tandem repeats; MLVA type: Multiple locus variable number tandem repeat analysis type; SIT: Spoligo International Type; MFI: Median fluorescence intensity; WGS: Whole genome sequencing; S: Streptomycin, anti-TB drug; I: Isoniazid, anti-TB drug; R: Rifampicin, anti-TB drug; E: Ethambutol, anti-TB drug; Km: Kanamycin, anti-TB drug; Am: Amikacin, anti-TB drug; Cm: Capreomycin, anti-TB drug; Eto: Ethionamide, anti-TB drug.

\section{Competing interests}

The authors declare that they have no competing interests.

\section{Authors' contributions}

RAN, CS, DS, PK, RAS, SP and EB designed the study. IB, RA and SS coordinated the project. AS performed the MLPA experiments. KT participated in the design of the MLPA probes. SS, IB and RA analyzed the MLPA data. NBA, NBZ, NTA and MA collected patient samples, isolated DNA and performed DST for first and second line drugs and GenoTypeMTBDRplus. NBA, NBZ, MA, NTA, NTU and RAS analyzed data obtained from DST and GenoTypeMTBDRplus. JB and DS performed MIRU-VNTR typing. EA and CS performed spoligotyping and analyzed the data. SS, NBZ, IB and RAN compiled all the data from the various methods and compared the data. SS, NBZ, IB and RAN wrote the first draft of the manuscript. All authors have read and approved the manuscript.

\section{Acknowledgments}

We would like to thank Gonnie Spierings at Luminex BV and MRC Holland for continuous support with the MLPA assay development. We thank Rina de Zwaan of the RIVM for technical assistance. This project was funded by the Dutch government through the Netherlands Organization for Health Research and Development (ZonMW) and the WOTRO Science for Global Development program, project nr 205100005

\section{Author details}

${ }^{1}$ KIT Biomedical Research, Royal Tropical Institute, Meibergdreef 39, 1105 AZ Amsterdam, The Netherlands. ${ }^{2}$ National TB Reference Laboratory, National Center for Tuberculosis and Lung Diseases, 50 Maruashvili Street, 0101 Tbilisi, Georgia. ${ }^{3}$ Instituto Venezolano de Investigaciones Cientificas (IVIC), Laboratorio de Genética Molecular, CMBC, Caracas, Venezuela. ${ }^{4}$ Institute of
Genetics and Microbiology, UMR 8621 CNRS/UPS11, Buildings 400 et 409, Faculty of Sciences - University Paris-Sud 11, 15, rue Georges Clémenceau, 91405 Orsay, France. ${ }^{5}$ Tuberculosis Reference Laboratory, Centre for Infectious Disease Control, Centre for Infectious Disease Research

Diagnostics and Perinatal Screening, National Institute for Public Health and the Environment, P.O. Box 1, 3720 BA Bilthoven, The Netherlands. ${ }^{6}$ National Reference Laboratory, National Center for Tuberculosis Problems, Ministry Health of the Republic Kazakhstan, 5, Bekhozhin str, Almaty 050010, Republic of Kazakhstan. ${ }^{7}$ MRC-Holland, Willem Schoutenstraat 6, 1057 DN Amsterdam, The Netherlands. ${ }^{8}$ National Center of Infectious and Parasitic Diseases, 26 Yanko Sakazov blvd, Sofia 1504, Bulgaria. ${ }^{9}$ Tuberculosis Reference Laboratory, Centre for Infectious Disease Control, National Institute for Public Health and the Environment, P.O. Box 1, 3720 BA Bilthoven, The Netherlands.

${ }^{10}$ Department of Pulmonary Disease, University Centre for Chronic Diseases, and Department of Medical Microbiology, Radboud University Nijmegen Medical Centre, P.O. Box 9101, 6500 HB Nijmegen, Netherlands.

Received: 18 February 2014 Accepted: 19 June 2014

Published: 7 July 2014

\section{References}

1. Borgdorff MW, van Soolingen D: The re-emergence of tuberculosis: what have we learnt from molecular epidemiology? Clin Microbiol Infect 2013, 19:889-901.

2. Devaux I, Kremer K, Heersma H, van Soolingen D: Clusters of multidrugresistant Mycobacterium tuberculosis cases, Europe. Emerg Infect Dis 2009, 15:1052-1060.

3. Dalla Costa ER, Lazzarini LC, Perizzolo PF, Diaz CA, Spies FS, Costa LL, Ribeiro AW, Barroco C, Schuh SJ, da Silva Pereira MA, Dias CF, Gomes HM, Unis G, Zaha A, da Silva PE A, Suffys PN, Rossetti ML: Mycobacterium tuberculosis of the RDRio genotype is the predominant cause of tuberculosis and associated with multidrug resistance in Porto Alegre City South Brazil. J Clin Microbiol 2013, 51:1071-1077.

4. Monteserin J, Camacho M, Barrera L, Palomino JC, Ritacco V, Martin A: Genotypes of Mycobacterium tuberculosis in patients at risk of drug resistance in Bolivia. Infect Genet Evol 2013, 17:195-201.

5. Gagneux S, DeRiemer K, Van T, Kato-Maeda M, de Jong BC, Narayanan S, Nicol M, Niemann S, Kremer K, Gutierrez MC, Hilty M, Hopewell PC, Small PM: Variable host-pathogen compatibility in Mycobacterium tuberculosis. Proc Natl Acad Sci U S A 2006, 103:2869-2873.

6. Comas I, Gagneux S: The past and future of tuberculosis research. PLoS Pathog 2009, 5:e1000600.

7. Ramaswamy S, Musser JM: Molecular genetic basis of antimicrobial agent resistance in Mycobacterium tuberculosis: 1998 update. Tuber Lung Dis 1998, 79:3-29.

8. Hershberg R, Lipatov M, Small PM, Sheffer H, Niemann S, Homolka S, Roach JC, Kremer K, Petrov DA, Feldman MW, Gagneux S: High functional diversity in Mycobacterium tuberculosis driven by genetic drift and human demography. PLoS Biol 2008, 6:e311.

9. Comas I, Homolka S, Niemann S, Gagneux S: Genotyping of genetically monomorphic bacteria: DNA sequencing in Mycobacterium tuberculosis highlights the limitations of current methodologies. PLoS One 2009, 4:e7815.

10. Bergval I, Sengstake S, Brankova N, Levterova V, Abadia E, Tadumaze N, Bablishvili N, Akhalaia M, Tuin K, Schuitema A, Panaiotov S, Bachiyska E, Kantardjiev T, De Zwaan R, Schürch AC, Van Soolingen D, Van't Hoog A, Cobelens F, Aspindzelashvili R, Sola C, Klatser P, Anthony R: Combined species identification, genotyping, and drug resistance detection of Mycobacterium tuberculosis cultures by MLPA on a bead-based array. PLoS One 2012, 7:e43240.

11. Bergval IL, Vijzelaar RN, Dalla Costa ER, Schuitema AR, Oskam L, Kritski AL, Klatser PR, Anthony RM: Development of multiplex assay for rapid characterization of Mycobacterium tuberculosis. J Clin Microbiol 2008, 46:689-699.

12. Stucki D, Gagneux S: Single nucleotide polymorphisms in Mycobacterium tuberculosis and the need for a curated database. Tuberc (Edinb ) 2013 , 93:30-39.

13. Theelen W, Reijans M, Simons G, Ramaekers FC, Speel EJ, Hopman AH: A new multiparameter assay to assess HPV $16 / 18$, viral load and physical status together with gain of telomerase genes in HPV-related cancers. Int J Cancer 2010, 126:959-975. 
14. Pham TD, Tran Vu TN, Tran TC, Loden M, Tuin K, Campbell Jl, Van Minh HN, Voong VP, Farrar JJ, Holt KE, Dougan G, Baker S: Identification of Salmonella enterica serovar Typhi genotypes by use of rapid multiplex ligation-dependent probe amplification. J Clin Microbiol 2013, 51:2950-2958.

15. Deshpande A, Gans J, Graves SW, Green L, Taylor L, Kim HB, Kunde YA, Leonard PM, Li PE, Mark J, Song J, Vuyisich M, White PS: A rapid multiplex assay for nucleic acid-based diagnostics. J Microbiol Methods 2010, 80:155-163.

16. Konialis C, Savola S, Karapanou S, Markaki A, Karabela M, Polychronopoulou S, Ampatzidou M, Voulgarelis M, Viniou NA, Variami E, Koumarianou A, Zoi K, Hagnefelt B, Schouten JP, Pangalos C: Routine application of a novel MLPA-based first-line screening test uncovers clinically relevant copy number aberrations in haematological malignancies undetectable by conventional cytogenetics. Hematology 2014, 19:217-24.

17. Blay P, Santamaria I, Pitiot AS, Luque $M$, Alvarado MG, Lastra A, Fernandez $Y$, Paredes A, Freije JM, Balbin M: Mutational analysis of BRCA1 and BRCA2 in hereditary breast and ovarian cancer families from Asturias (Northern Spain). BMC Cancer 2013, 13:243.

18. Joosten SA, Goeman JJ, Sutherland JS, Opmeer L, de Boer KG, Jacobsen M, Kaufmann SH, Finos L, Magis-Escurra C, Ota MO, Ottenhoff TH, Haks MC: Identification of biomarkers for tuberculosis disease using a novel dual-color RT-MLPA assay. Genes Immun 2012, 13:71-82.

19. Alland D, Whittam TS, Murray MB, Cave MD, Hazbon MH, Dix K, Kokoris M, Duesterhoeft A, Eisen JA, Fraser CM, Fleischmann RD: Modeling bacterial evolution with comparative-genome-based marker systems: application to Mycobacterium tuberculosis evolution and pathogenesis. J Bacteriol 2003, 185:3392-3399.

20. Stucki D, Malla B, Hostettler S, Huna T, Feldmann J, Yeboah-Manu D, Borrel S, Fenner L, Comas I, Coscolla M, Gagneux S: Two new rapid SNP-typing methods for classifying Mycobacterium tuberculosis complex into the main phylogenetic lineages. PLOS One 2012, 7:e41253.

21. Tuberculosis surveillance and monitoring in Europe 2013. http://www ecdc.europa.eu/en/publications/Publications/Tuberculosis-surveillancemonitoring-2013.pdf

22. Shubladze N, Tadumadze N, Bablishvili N: Molecular patterns of multidrug resistance of in Georgia. Int J Mycobacteriol 2013, 2:73-78.

23. Tukvadze N, Kempker RR, Kalandadze I, Kurbatova E, Leonard MK, Apsindzelashvili R, Bablishvili N, Kipiani M, Blumberg HM: Use of a molecular diagnostic test in AFB smear positive tuberculosis suspects greatly reduces time to detection of multidrug resistant tuberculosis. PLoS One 2012, 7:e31563.

24. Tukvadze N, Bablishvili N, Apsindzelashvili R, Blumberg HM, Kempker RR: Performance of the MTBDRsI assay in Georgia. Int J Tuberc Lung Dis 2014 18:233-239

25. Pardini M, Niemann S, Varaine F, lona E, Meacci F, Orru G, Yesilkaya H, Jarosz T, Andrew P, Barer M, Checchi F, Rinder H, Orefici G, Rusch-Gerdes S, Fattorini L, Oggioni MR, Bonnet M: Characteristics of drug-resistant tuberculosis in Abkhazia (Georgia), a high-prevalence area in Eastern Europe. Tuberc (Edinb) 2009, 89:317-324.

26. Niemann S, Diel R, Khechinashvili G, Gegia M, Mdivani N, Tang YW: Mycobacterium tuberculosis Beijing lineage favors the spread of multidrug-resistant tuberculosis in the Republic of Georgia. J Clin Microbiol 2010, 48:3544-3550.

27. Bonnet M, Pardini M, Meacci F, Orru G, Yesilkaya H, Jarosz T, Andrew PW, Barer M, Checchi F, Rinder H, Orefici G, Rusch-Gerdes S, Fattorini L, Oggion MR, Melzer J, Niemann S, Varaine F: Treatment of tuberculosis in a region with high drug resistance: outcomes, drug resistance amplification and re-infection. PLoS One 2011, 6:e23081

28. Gardner SN, Thissen JB, McLoughlin KS, Slezak T, Jaing CJ: Optimizing SNP microarray probe design for high accuracy microbial genotyping. J Microbiol Methods 2013, 94:303-310.

29. Schürch AC, Kremer $K$, Hendriks AC, Freyee B, McEvoy CR, van Crevel $R$, Boeree MJ, van Helden HP, Warren RM, Siezen RJ, Van Soolingen D: SNP/RD typing of Mycobacterium tuberculosis Beijing strains reveals local and worldwide disseminated clonal complexes. PLoS One 2011, 6:e28365.

30. Hornstra HM, Priestley RA, Georgia SM, Kachur S, Birdsell DN, Hilsabeck R, Gates LT, Samuel JE, Heinzen RA, Kersh GJ, Keim P, Massung RF, Pearson T: Rapid typing of Coxiella burnetii. PLOS One 2011, 6:e26201.

31. Leekitcharoenphon P, Kaas RS, Thomsen MC, Friis C, Rasmussen S, Aarestrup FM: snpTree-a web-server to identify and construct SNP trees from whole genome sequence data. BMC Genomics 2012, 13(Suppl 7):S6
32. Gomgnimbou MK, Hernandez-Neuta I, Panaiotov S, Bachiyska E, Palomino JC, Martin A, Del PP, Refregier G, Sola C: Tuberculosis-Spoligo-RifampinIsoniazid Typing: an All-in-One Assay Technique for Surveillance and Control of Multidrug-Resistant Tuberculosis on Luminex Devices. J Clin Microbiol 2013, 51:3527-3534.

33. Demay C, Liens B, Burguiere T, Hill V, Couvin D, Millet J, Mokrousov I, Sola C, Zozio T, Rastogi N: SITVITWEB-a publicly available international multimarker database for studying Mycobacterium tuberculosis genetic diversity and molecular epidemiology. Infect Genet Evol 2012, 12:755-766.

34. Kamerbeek J, Schouls L, Kolk A, van Agterveld M, van Soolingen D, Kuijper S, Bunschoten A, Molhuizen H, Shaw R, Goyal M, Van Embden JD: Simultaneous detection and strain differentiation of Mycobacterium tuberculosis for diagnosis and epidemiology. J Clin Microbiol 1997 35:907-914

35. van Embden JD, Cave MD, Crawford JT, Dale JW, Eisenach KD, Gicquel B, Hermans P, Martin C, McAdam R, Shinnick TM: Strain identification of Mycobacterium tuberculosis by DNA fingerprinting: recommendations for a standardized methodology. J Clin Microbiol 1993, 31:406-409.

36. Bourgey M, Lariviere M, Richer C, Sinnett D: ALG: automated genotype calling of Luminex assays. PLoS One 2011, 6:e19368.

37. Jugheli L, Bzekalava N, de Rijk P, Fissette K, Portaels F, Rigouts L: High level of cross-resistance between kanamycin, amikacin, and capreomycin among Mycobacterium tuberculosis isolates from Georgia and a close relation with mutations in the rrs gene. Antimicrob Agents Chemother 2009, 53:5064-5068.

38. Streicher EM, Bergval I, Dheda K, Bottger EC, Gey van Pittius NC, Bosman M, Coetzee G, Anthony RM, van Helden PD, Victor TC, Warren RM: Mycobacterium tuberculosis population structure determines the outcome of genetics-based second-line drug resistance testing. Antimicrob Agents Chemother 2012, 56:2420-2427.

39. Shamputa IC, Jugheli L, Sadradze N, Willery E, Portaels F, Supply P, Rigouts $L$ : Mixed infection and clonal representativeness of a single sputum sample in tuberculosis patients from a penitentiary hospital in Georgia. Respir Res 2006, 7:99

40. Liao Y, Wang X, Sha C, Xia Z, Huang Q, Li Q: Combination of fluorescence color and melting temperature as a two-dimensional label for homogeneous multiplex PCR detection. Nucleic Acids Res 2013, 41:e76.

41. Abadia E, Zhang J, dos Vultos T, Ritacco V, Kremer K, Aktas E, Matsumoto T, Refregier G, van Soolingen D, Gicquel B, Sola C: Resolving lineage assignation on Mycobacterium tuberculosis clinical isolates classified by spoligotyping with a new high-throughput 3R SNPs based method. Infect Genet Evol 2010, 10:1066-1074.

42. Mokrousov I: The quiet and controversial: Ural family of Mycobacterium tuberculosis. Infect Genet Evol 2012, 12:619-629.

43. Supply P, Allix C, Lesjean S, Cardoso-Oelemann M, Rusch-Gerdes S, Willery E, Savine E, de Haas P, van Deutekom H, Roring S, Bifani P, Kurepina N, Kreiswirth B, Sola C, Rastogi N, Vatin V, Gutierrez MC, Fauville M, Niemann S, Skuce R, Kremer K, Locht C, van Soolingen D: Proposal for standardization of optimized mycobacterial interspersed repetitive unit-variable-number tandem repeat typing of Mycobacterium tuberculosis. J Clin Microbiol 2006, 44:4498-4510.

44. de Beer JL, Akkerman OW, Schurch AC, Mulder A, van der Werf TS, van der Zanden AG, van Ingen J, van Soolingen D: Optimization of standard in-house 24-locus variable-number tandem-repeat typing for Mycobacterium tuberculosis and its direct application to clinical material. J Clin Microbiol 2014, 52:1338-1342.

45. Allix-Beguec $C$, Harmsen D, Weniger T, Supply P, Niemann S: Evaluation and strategy for use of MIRU-VNTRplus, a multifunctional database for online analysis of genotyping data and phylogenetic identification of Mycobacterium tuberculosis complex isolates. J Clin Microbiol 2008, 46:2692-2699

doi:10.1186/1471-2164-15-572

Cite this article as: Sengstake et al: Optimizing multiplex SNP-based data analysis for genotyping of Mycobacterium tuberculosis isolates. BMC Genomics 2014 15:572. 